https://creativecommons.org/licenses/by/4.0/

\title{
LA ACROFOBIA Y SU TRATAMIENTO A TRAVÉS DE REALIDAD VIRTUAL
}

\section{Acrophobia and its treatment through virtual reality}

\author{
AARÓN RAMÍREZ*, SANDRA JOHANNA BENÍTEZ**, MARÍA ANGÉLICA GÓMEZ***
}

Recibido: 19 de diciembre de 2016. Aceptado: 29 de diciembre de 2016

DOI: http://dx.doi.org/10.21017/rimci.2017.v4.n7.a24

\begin{abstract}
Resumen
La acrofobia es un miedo irracional, exagerado e irreprimible a las alturas, las personas que la padecen pueden llegar a sentir temor no solamente desde grandes alturas, sino también al estar en un balcón, unas escaleras, entre otras. En la actualidad existen diferentes tipos de tratamientos como lo son los psicológicos, los de exposición, los cognitivoscomportamentales, donde se ha considerado la exposición como la manera más efectiva de afrontar estar fobia, sin embargo, la realidad virtual se ha venido convirtiendo en una de las técnicas más utilizadas e innovadoras porque ofrece entornos virtuales donde se cuenta con la ventaja de poder controlar y personalizar el ambiente dependiendo de la necesidad del paciente, por tanto promete ser una buena manera de llevar a cabo la terapia de exposición, ya que permite proporcionar sensaciones y la idea general de estar presente en una situación temida donde la persona no se encontrará en ningún momento en peligro físico y podrá interactuar con los elementos del ambiente a través de diferentes herramientas que contribuyen a que la realidad virtual sea todo un éxito.
\end{abstract}

Palabras clave: miedo a las alturas, realidad virtual, exposición virtual, plataforma.

\begin{abstract}
Acrophobia is an irrational fear, exaggerated and irrepressible to the heights, people who suffer from it can become afraid not only from great heights, but also from being on a balcony, stairs, among others. Nowadays there are different types of treatments such as psychological, exposure, cognitive-behavioral, where exposure has been considered as the most effective way to face this phobia, however, virtual reality has been becoming one of the most used and innovative techniques, because it offers virtual environments where advantage such as been able to control and customize the environment depending on the need of the patient. Therefore promises to be a good way to carry out the exposure therapy, since it allows you to provide sensations and the general idea of being present in a dreaded situation where the person will not be at any time in physical danger and will be able to interact with the elements of diferent environments through different tools that contribute that virtual reality is a success.
\end{abstract}

Keywords: fear of heights, virtual reality, virtual expo, platform, stage.

\section{INTRODUCCIÓN}

U NA PERSONA que sufre de acrofobia puede llegar a presentar diferentes síntomas fisiológicos como lo son los mareos, taquicardia, parálisis, sudoración, ansiedad entre otras. Así mismo para algunos este tipo de sensaciones pueden presentarse con solo pensar en una situación de peligro.
Para ello existen diferentes formas de tratarla como los tratamientos psicológicos que se reducen básicamente al psicoanálisis, las terapias cognitivocomportamentales empleadas en combinación con estrategia a las terapias de exposición que van destinadas a cambiar los patrones de pensamiento, insistiendo en la diferencia entre pensamientos realistas e irrealistas o la diferencia entre posible y

\footnotetext{
* Ingeniero de Sistemas de la Escuela Colombiana de Ingeniería, Diplomado en Gerencia de Proyectos del Politécnico de Colombia, Certificación en Negociación - Negotiation Camp Institute, estudiante de Maestría en Gestión de Proyectos (Universidad EAN - Université du Québec à Chicoutimi). Correo electrónico: aramirez@urepublicana.edu.co

** Estudiante de décimo semestre de Ingeniería de sistemas de la Corporación Universitaria Republicana. Correo electrónico: johanna. benitez18@gmail.com

***Estudiante de décimo semestre de Ingeniería de sistemas de la Corporación Universitaria Republicana. Correo electrónico: mgangelik84@gmail.com
} 
probable las cuales han demostrado ser eficaces, pero dada la naturaleza de estos trastornos o miedos suelen ser costosas en términos de tiempo, dinero y esfuerzos, por último se encontró la realidad virtual la cual actualmente constituye una de las tecnologías más emergentes que permite generar ambientes tridimensionales, simulando la realidad, es por ello que se ha convertido en los últimos años en una herramienta de trabajo muy útil para las ciencias de la salud, tanto en la investigación básica como en la aplicada, una de las áreas donde ha tenido gran impacto han sido las psicológicas ya que ha empezado a ser una de las técnicas más utilizadas y novedosas en el tratamiento de trastornos psicológicos como lo son las fobias.

En este ámbito la idea básica es insertar al usuario dentro de un ambiente imaginario (mundo virtual) utilizando herramientas como lo son el casco de realidad virtual con capacidad de recrear los cincos sentidos, traje XTAL VICION que es un visor que combina imágenes en movimiento y que es capaz de reflejarlas a distancia en prendas de ropa además de que crea la ilusión de la transparencia de la materia y de la apariencia de invisibilidad para la cual emplean una técnica denomina Camuflaje óptico y se basa en una idea muy simple: si se proyecta una imagen de lo que está detrás de una persona sobre la ropa que viste en ese momento, la ilusión que se crea es que el cuerpo de esa persona es transparentes, gafas en 3D las cuales no dejan de ser sorprendentes, estas gafas tienen muchas formas de poder mostrarnos lo que estamos viendo y así generar un ambiente seguro donde se pueda conmutar de ambientes con plena seguridad, la persona no se encontrará en ningún momento en riesgo físico y podrán interactuar con los elementos del medio virtual con ventajas frente a la tradicional exposición en vivo como lo son economía, control y personalización del ambiente.

En el año 1956 Morton Heilig, inventó la primera máquina de realidad virtual aunque no la patentó hasta 1962. Se llamaba Sensorama y combinaba vídeo 3D a todo color, audio, vibraciones, viento y olores. Contaba sólo con 5 grabaciones de películas de dos minutos. Esta tecnología se empezó a implementar en el tratamiento de fobias desde los años 60 aunque fue hasta 1992 que empezó a utilizarse en los centros de psicología cuando el grupo liderado por Max North de la Clark Atlanta University (CAU)
(North, North y Coble, 1996) descubrió, fruto de la casualidad, que los ambientes de realidad virtual eran capaces de inducir una alta activación emocional en pacientes diagnosticados de trastornos fóbicos [1]. En Estados Unidos esta técnica ha venido siendo utilizada desde hace varios años, sin embargo en Colombia aún no es muy conocida.

Estudios como los realizados por la Universidad de Ámsterdam en personas que tenían miedo a las alturas desveló que el tratamiento con Realidad Virtual es tan eficaz como la exposición en vivo a las situaciones fóbicas, pero más seguro, y en la intimidad de una consulta de Psicología [2].

Existe también una noticia periodística (cyberdge) acerca de un estudio llevado a cabo por el Grupo Médico Káiser-Permanente de California en el que se utilizó realidad virtual para el tratamiento de acrofobia en 1994. Los 32 pacientes sobre los que se realizó el estudio debían de atravesar un profundo barranco pasando por un puente colgante y una tabla de madera, obteniendo un porcentaje de éxito del $90 \%$.

Ha habido otros estudios como los realizados por Rothbaum y grupos del Norte de la universidad de Clark Atlanta, además, Choi Jack, Ku, Shin y Kim (2001) y Jang, Ku, Choi, Wiederhold, Nam, Kim y Kim (2002) quienes también han demostrado que la técnica de exposición a través de realidad virtual ha sido eficaz en el tratamiento de la acrofobia.

Los miembros del Departamento de Psicología Básica, Clínica y Psicología de la Universidad Jaime I, en Castellón, piensan que las herramientas basadas en la realidad virtual pueden ofrecer una manera muy útil para esta clase de tratamientos; por ello han desarrollado terapias basadas en la realidad virtual aplicada a pacientes con claustrofobia, agorafobia, fobia a los insectos y miedo a volar [3].

\section{MÉtodo}

\section{A. Definición de parámetros}

Este proyecto propone ser una herramienta de apoyo en el tratamiento de la fobia a las alturas mediante la realidad virtual, el cual debe de ir de 
la mano con especialistas de la salud mental quienes deberán medir su efectividad a través de herramientas que permitan medir signos vitales, nivel de sudoración, frecuencia cardiaca, frecuencia de la voz, entre otros que ellos consideren.

Para dar desarrollo al proyecto se llevó a cabo una serie de procedimientos que se enumeran a continuación:

1. Se estableció un cronograma con las actividades a desarrollar y los tiempos de ejecución de cada una de ellas, hasta llegar al producto final.

2. Se establecen que recursos humanos y financieros son necesarios para generar el ambiente virtual y poderlo vender al usuario final, en este caso, áreas de la psicología orientadas al tratamiento de la fobia a las alturas.

3. Para determinar la fobia a la cual iría orientado el escenario virtual se realizó inicialmente una encuesta a 100 personas ubicadas en la ciudad de Bogotá D.C, Colombia, esto con el fin de:

- Identificar la fobia a tratar.

- Identificación de la población.

- Analizar y evaluar la información recaudada.

- Establecer un campo de desarrollo.

4. Se encuestó a un profesional de la salud especialista en el área de psicología y un psicólogo en formación, con el fin de:

- Identificar la viabilidad del proyecto que se desea implementar como apoyo al tratamiento de la fobia a las alturas, según los datos obtenidos en la encuesta inicial.

- Conocer de qué manera se está tratando esta fobia actualmente en Colombia.

5. Para crear un mundo virtual completo, se requiere tener: un software de creación de escenarios en 3D, un sistema de captura de movimientos en tiempo real, y unas gafas de realidad virtual que permitan al usuario experimentar ese nuevo universo inventado. Todas estas herramientas existen desde hace años, pero sólo ahora ofrecen la potencia y versatilidad suficientes para trabajar en equipo y, aún más importante, en tiempo real, para lo cual se generó una discusión acerca de cuál sería la mejor plataforma para desarrollar el ambiente virtual a implementar a través de las gafas 3D de la marca VR BOX, decidiéndonos por Unity que es un motor de videojuego multiplataforma creado por Unity Technologies.

6. Se define el diseñó del escenario deseado para empezarlo a implementar, ejecutar y así realizar su debido seguimiento para las posteriores mejoras.

\section{B. Descripción del escenario}

El proyecto se desarrollará en Unity por ser multiplataforma, por tanto, está disponible como una herramienta de desarrollo para Windows, OSX, otra característica importante es que cuenta con soporte de compilación. En el proyecto se propone un escenario principal basado en dos edificios ubicados en un terreno rodeado por árboles y poco montañoso, por tanto, se contará con el efecto del viento, los edificios estarán comunicados entre sí con una tabla para que la persona se pueda desplazar del uno al otro, en cada uno de los pisos del edificio se encontraran ubicada una tabla, con el fin de que el paciente comience interactuando desde un piso bajo y a medida de que se vayan evidenciando resultados se incremente gradualmente la altura, adicional a esto se tendrá una superficie acolchada ubicada en la parte inferior, es decir, sobre el piso esto con la finalidad de que el paciente tenga una sensación de seguridad y en caso de caer de alguna de las tablas fuera a la superficie acolchada donde rebotaría. Figura 1.

La escala de unidades de medida aplicada fue la predeterminada por Unity, cuando se aplica rigidbody a los objetos es 1 unidad $=1$ metro, entonces la escala de su mesh importado se mantiene [4].

Teniendo esto en cuenta, podemos decir que las unidades de medida (las proporciones físicas / 


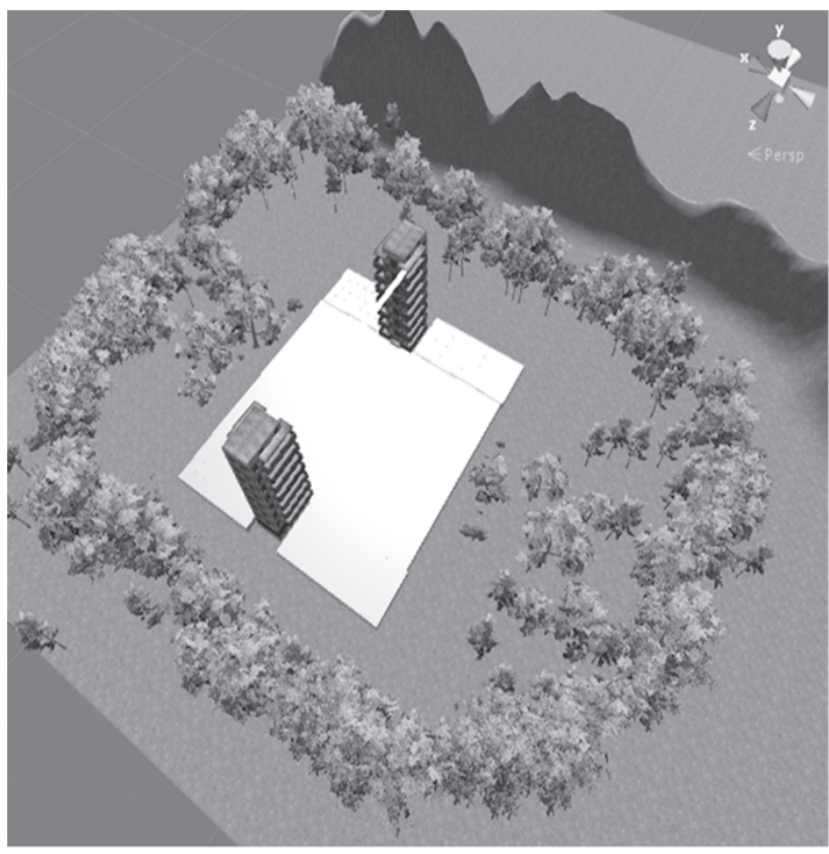

Fig. 1. Perspectiva completa del escenario.

geométricas) de un proyecto en Unity trabajan con unidades que cada cual a su gusto determinan y que significan las mismas, por tanto, pueden ser tomadas como metros, centímetros, kilómetros, pulgadas, pies, pársecs, angstroms, etc. Es decir, al considerar un objeto de escala $(x, y, z)=(1,1$, 2 ) entonces suponiendo que tomamos las unidades como metros, el objeto va a medir en el eje $X$ 1 metro, en el eje $Y 1$ metro y en el eje Z 2 metros, $\mathrm{y}$ tomando la posición con respecto al origen de coordenadas de la escena como $(x, y, z)=(2.5$, $3.5,4.5)$ esto significará que el objeto estará a $2.5 \mathrm{~m}$ en $X$ del origen, a $3.5 \mathrm{~m}$ en $Y$ del origen y a $4.5 \mathrm{~m}$ en $\mathrm{Z}$ del origen [5]. Figura 2.

\section{Proyección}

Existen varios autores que resaltan la efectividad de la realidad virtual en el tratamiento de las fobias, entre ellos esta Iván Alsina psicólogo y profesor universitario, especialista en el uso y aplicaciones de las TIC en psicología, salud mental, y educación, por lo que nos habla de VirtualRet, plataforma virtual de atención de ansiedad y fobia y de las bases de la nueva psicología que nace en el año 2012 debido al trabajo en equipo de expertos en Psicología Clínica y profesionales de la Tecnología, con el ánimo de hacer llegar las ventajas que

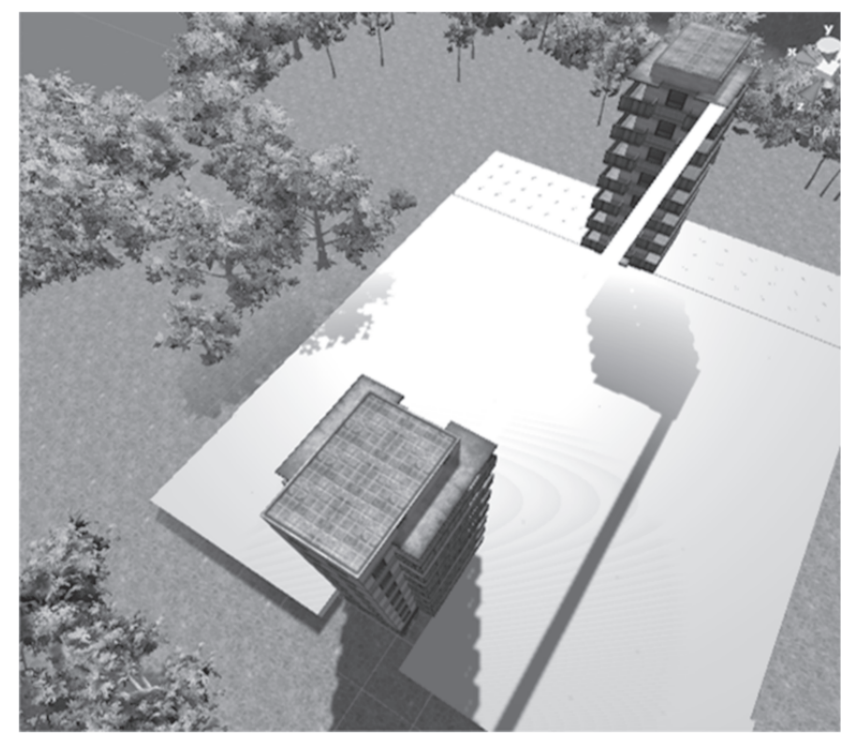

Fig. 2. Ambiente virtual.

ofrecía la realidad virtual a los profesionales del campo de la salud mental, afirmando que la técnica era basada en un entorno que día tras día iba en auge a consecuencia de los avances tecnológicos y que a pesar de que contaban con el acceso a dicha tecnología, en la ciudad donde residía era un campo en el que pocos habían explorado y para él darlo a conocer en el sector de la salud era un reto que quería lograr.

Por otro lado Joaquín Cantó, profesor del Instituto de Ciencias de la Educación de Alicante (España), psicólogo y master en psicología de la salud le apuesta todo a este método de realidad virtual, quien dice afirmar «es una terapia, con ellas trasladamos al paciente al entorno adecuado a sus necesidades y podemos ir controlando el grado de exposición al mismo».

Y como bien lo dice Cantó es una terapia moderna, la cual busca revolucionar, innovar y acortar esos tratamientos extensos y de alto costo. Con esta nueva herramienta de apoyo en el campo de la salud en Bogotá se quiere brindar estos beneficios, con pocos recursos y eficaces [6].

El objetivo principal de este trabajo es diseñar un escenario como herramienta de apoyo en el tratamiento del miedo a las alturas mediante realidad virtual obteniendo una excelente satisfacción en el sector de la salud, el cual está compuesto prin- 
cipalmente por psicólogos y especialistas en el tratamiento de las fobias (acrofobia); quienes comentan que por una parte esta herramienta les puede ser de gran apoyo en el momento de tratar los pacientes que sufren de esta fobia, los costos podrían bajar notablemente al igual que el tiempo en cada terapia o tratamiento.

Nuestro proyecto finalizaría con la implementación de la herramienta inicialmente en unos 10 centros psicológicos con el fin de comprobar su efectividad y determinar si el ambiente virtual creado es adecuado en el tratamiento del miedo a las alturas.

De igual manera con los resultados obtenidos de la prueba realizada por los diferentes centros se pretende generar mejoras a la herramienta de apoyo con el fin de que más centros médicos vean en nosotros una oportunidad de hacer un tratamiento más preciso a sus pacientes pudiendo vender nuestro producto y contribuir a la salud.

\section{Discusión}

La realidad virtual ha venido tomando fuerza, debido a que los proyectos que implementan este método han demostrado un alto índice de eficacia. Pueden llegar a abarcar cualquier situación de la vida cotidiana puesto que esta tiene la capacidad de que un ser humano tenga la sensación de encontrase en un entorno real pero en realidad está actuando en un mundo virtual y lo mejor de todo es que permite que el usuario interactué en tiempo real.

Otra de las ventajas con las que nos encontramos es que este mundo de Realidad Virtual (RV) es controlado estrictamente por nosotros, quienes somos los que le programamos y decimos que debe hacer. No se necesita salir y exponer al paciente para tratarle la fobia de la cual padece, adicional permite realizar el procedimiento o terapia las veces que se considere necesario a muy bajo costo y obteniendo los mismos o incluso mejores resultados a de los procedimiento clásicos [7].

Psious empres nació en julio de 2013, quien se dedica en la actualidad a implementar la realidad virtual en el sector de la salud, consiguió una inversión de un millón de euros para el tratamiento de varias fobias con simulación en 3D. Esta gran compañía lo integran un grupo de 21 personas, entre las que se pueden destacar a, psicólogos, ingenieros, diseñadores, videojugadores, especialistas en 3D e informáticos. Su modelo de negocio es alcanzar facturación internacional en un $60 \%$ con la venta de esta aplicación [8].

\section{Conclusiones}

La exposición mediante realidad virtual ha demostrado ser eficaz para el tratamiento del miedo a las alturas, ya que se han realizado diversos estudios que han demostrado su eficacia.

Muchas investigaciones realizadas sobre el tratamiento de las fobias con realidad virtual son controvertidas debido a que los resultados pueden producir un gran avance para algunas partes o una gran inconformidad para otras.

Algunos centros médicos podrían interesarse en nuestro producto y de acuerdo a los resultados generados implementarlo de manera permanente, para lo cual se debe de garantizar las actualizaciones necesarias y así estar a la vanguardia del avance de las TICs, por ejemplo cada vez hay un rol cada vez mayor de las tecnologías móviles.

La realidad virtual ha ayudado a las TICs a encontrar un espacio de trabajo significativo a lo que refiere a la salud.

\section{REFERENCIAS}

[1] I. Alsina. Entornos de Realidad Virtual para combatir la ansiedad. (s. f). Universidad Oberta de Catalunya. Mosaic. Disponible en: http:/ / mosaic. uoc.edu/2013/10/31/entornos-de-realidad-virtual-para-combatir-la-ansiedad/. 2013.

[2] El Prado psicólogos. Realidad virtual: eficacia e innovación en el tratamiento de las fobias y miedos. Disponible en: http://www. elpradopsicologos. es/terapia/realidad-virtual/

[3] A. Bartolomé. Realidad virtual, una herramienta eficaz para tratar las fobias. CuidatePlus. Disponible en: http:/ / www.dmedicina.com/enfermedades/psiquiatricas/2003/12/10/realidad-virtual- herramienta-eficaz-tratar-fobias3713.html. 2013. 
[4] Unity Documentation. Disponible en: https:// docs.unity3d.com/es/current/Manual/classRigidbody.html. 2016.

[5] [Unity 3D] Tutorial para novatos (2 - Objetos). Olivera, J. 08 de abril de 2013. Disponible en: https:/ / alittlebigof.wordss.com/2013/04/08/ unity-3d-tutorial-para-novatos-2-objetos /

[6] A. Pino. Realidad virtual contra las fobias. Disponible en: http://www.diarioinformacion. com/ alicante/2016/10/09/realidad-virtual-fobias / 1814664.html. 2016.

[7] Previ. (s.f). Disponible en: http://www.previsl. com/ver/79/realidad-virtual.html/

[8] LaVangurdia. (s.f). Disponible en: http://www. lavanguardia.com/economia/pymes/20160202/ 301841591008/psious-tratamiento-fobias.html 